\title{
Synchronization in Network Structures: Entangled Topology as Optimal Architecture for Network Design
}

\author{
Luca Donetti $^{1,3}$, Pablo I. Hurtado ${ }^{1,2}$, and Miguel A. Muñoz ${ }^{1}$ \\ ${ }^{1}$ Departamento de Electromagnetismo y Física de la Materia, and Instituto Carlos I \\ de Física Teórica y Computacional \\ Facultad de Ciencias, Universidad de Granada, 18071 Granada, Spain \\ ${ }^{2}$ Laboratoire des Colloïdes, Verres et Nanomatériaux, \\ Université Montpellier II, Montpellier 34095, CEDEX 5 France \\ ${ }^{3}$ Departamento de Electrónica y Tecnología de Computadores \\ Facultad de Ciencias, Universidad de Granada, 18071 Granada, Spain \\ donetti@ugr.es, phurtado@onsager.ugr.es, mamunoz@onsager.ugr.es
}

\begin{abstract}
In these notes we study synchronizability of dynamical processes defined on complex networks as well as its interplay with network topology. Building from a recent work by Barahona and Pecora [Phys. Rev. Lett. 89, 054101 (2002)], we use a simulated annealing algorithm to construct optimally-synchronizable networks. The resulting structures, known as entangled networks, are characterized by an extremely homogeneous and interwoven topology: degree, distance, and betweenness distributions are all very narrow, with short average distances, large loops, and small modularity. Entangled networks exhibit an excellent (almost optimal) performance with respect to other flow or connectivity properties such as robustness, random walk minimal firstpassage times, and good searchability. All this converts entangled networks in a powerful concept with optimal properties in many respects.
\end{abstract}

\section{Introduction}

It is broadly recognized that most complex systems in Nature are organized as intricated network patterns [1,2]. This observation has triggered an intense research effort aimed at understanding the organizing principles of these networks, their structural properties, and the interplay between topology and dynamics [1,2]. It was recently recognized that the classical models of random networks developed in graph theory were unable to describe the random but structured, hierarchical network patterns found in Nature. Since then, a number of paradigmatic models (as small-world and scale-free nets 2]) have seen the light. They mimic some of the striking properties observed in real complex networks. In any case, network structures play an important role in many contexts ranging from brain neural circuits, cellular function webs, ecosystems, social networks, food webs, etc., to power grids, Internet or the world wide web. While most of 
the initial effort was put into understanding the topological properties of networks, the interest has gradually shifted towards the analysis of the interplay between topology and the dynamics of network components. In general, each element (node) in a network undergoes a dynamical process while coupled to other nodes. The system collective behavior depends strongly on the efficiency of communication paths, which is in turn dictated by the underlying network topology. In this way, the network structure determines to a large extent the possibility of a coherent response.

Complete synchronization is the most prominent example of coherent behavior, and is a key phenomenon in systems of coupled oscillators as those characterizing most biological networks or physiological functions 3. For instance, synchronized neural firing has been suggested as specially relevant for neural signal transmission 4. From a more technological point of view, precision synchronization of computer clocks in local area networks and the Internet is essential for optimal network performance. Moreover, in an interesting twist, the dynamics toward synchronization has been recently used as a dynamical process unveiling the underlying community structure in complex networks [5].

Here we study how synchronous behavior is affected by the network structure. The range of stability of a synchronized state is a measure of the system ability to yield a coherent response and to distribute information efficiently among its elements, while a loss of stability fosters pattern formation [6]. Here we answer the following question: which is the topology that maximizes the network synchronizability? [7]. We will construct such optimal topologies, for any fixed number of nodes and links, by employing an optimization procedure. The resulting structures, that we call entangled networks, are optimal not only for synchronizability, but also regarding other flow or connectivity properties.

The paper is structured as follows. In section 2 we summarize the spectral approach to synchronization, following Ref. 6]. In section 3 we introduce the optimization procedure to obtain networks with optimal synchronizability. Section 4 discusses the relation between the emerging structures and other optimal network designs in the literature. Finally, conclusions and further developments are presented. A shorter presentation of this work has been published before [7].

\section{Spectral Approach to Synchronization in Networks}

Consider $N$ identical oscillators at the nodes of an undirected and unweighted graph. The state of an oscillator is represented in general by a vector $\mathbf{x}_{i}, i \in$ $[1, N]$, where $N$ is the number of nodes. The network is characterized by its Laplacian matrix $\mathbf{L}$, with elements $L_{i i}=k_{i}$ (the degree of node $i$ ), $L_{i j}=-1$ if nodes $i$ and $j$ are connected, and $L_{i j}=0$ otherwise. $\mathbf{L}$ is therefore a symmetric matrix with zero-sum rows and real, non-negative spectrum. The dynamics of the $i$-th node can then be represented in a very general form as,

$$
\frac{\mathrm{d} \mathbf{x}_{i}}{\mathrm{~d} t}=\mathbf{F}\left(\mathbf{x}_{i}\right)-\sigma \sum_{j=1}^{N} L_{i j} \mathbf{H}\left(\mathbf{x}_{j}\right)
$$


Here $\mathbf{F}(\mathbf{x})$ and $\mathbf{H}(\mathbf{x})$ are unspecified evolution and coupling functions, respectively. In particular, $\mathbf{F}(\mathbf{x})$ controls the dynamics of the uncoupled oscillators, while $\mathbf{H}(\mathbf{x})$ specifies how variables at different nodes couple together. Most dynamical processes studied in the literature regarding synchronization can be recasted in forms equivalent to eq. (10) (see [6] for more general couplings).

In the synchronized state all oscillators behave identically at all times. That is, $\mathbf{x}_{i}(t)=\mathbf{x}^{s}(t) \forall i \in[1, N]$, where $\mathbf{x}^{s}(t)$ is solution of the uncoupled equation $\dot{\mathbf{x}}^{s}=\mathbf{F}\left(\mathbf{x}^{s}\right)$ ( $\dot{\mathbf{x}}$ represents the time derivative of $\mathbf{x}$ ). The $N-1$ synchronization constraints $\mathbf{x}_{1}(t)=\mathbf{x}_{2}(t)=\ldots=\mathbf{x}_{N}(t)$ define a synchronization manifold. This manifold is invariant owing to the zero-sum row condition in the Laplacian matrix $\mathbf{L}$ 6. We are interested here in the stability of the synchronized state. For that, we introduce small perturbations $\xi_{i}$ such that $\mathbf{x}_{i}=\mathbf{x}^{s}+\xi_{i}$, and expand to first order to arrive at: $\dot{\xi}_{i}=\sum_{j=1}^{N}\left[\partial \mathbf{F}\left(\mathbf{x}^{s}\right) \delta_{i j}-\sigma L_{i j} \partial \mathbf{H}\left(\mathbf{x}^{s}\right)\right] \cdot \xi_{i}$, where $\partial \mathbf{M}$ stands for the Jacobian of a matrix $\mathbf{M}$. Diagonalization of $\mathbf{L}$ transforms these equations into a set of $N$ independent equations for the normal modes [6, 7]:

$$
\frac{\mathrm{d} \mathbf{y}_{k}}{\mathrm{~d} t}=\left[\partial \mathbf{F}\left(\mathbf{x}^{s}\right)-\sigma \lambda_{k} \partial \mathbf{H}\left(\mathbf{x}^{s}\right)\right] \cdot \mathbf{y}_{k}
$$

where $\lambda_{k}, k \in[1, N]$, are the eigenvalues of $\mathbf{L}, 0=\lambda_{1} \leq \lambda_{2} \leq \ldots \leq \lambda_{N}$. All the resulting equations have the same form $\dot{\mathbf{y}}=\left[\partial \mathbf{F}\left(\mathbf{x}^{s}\right)-\alpha \partial \mathbf{H}\left(\mathbf{x}^{s}\right)\right] \cdot \mathbf{y}$ for some positive constant $\alpha$. The synchronized state $\mathbf{x}^{s}$ will be stable if and only if all the perturbations fade away in time. This is equivalent to demanding the maximum Lyapunov exponent $\eta_{\max }(\alpha)$ associated with the normal modes orthogonal to the synchronization manifold to be negative. The function $\eta_{\max }(\alpha)$ has been called the master stability function in literature [6], and its dependence on $\alpha$ has an universal "V-shape" for most oscillating systems. In particular, $\eta_{\max }(\alpha)$ is negative only in an interval $\alpha_{A}<\alpha<\alpha_{B}$. The synchronized state will be stable if all the non-trivial eigenvalues of $\mathbf{L},\left\{\lambda_{k}: k=2, \ldots, N\right\}$, lie within the interval $\left[\alpha_{A} / \sigma, \alpha_{B} / \sigma\right]$. The following inequality then guarantees that there always exists a coupling strength $\sigma$ for which the synchronized state is stable,

$$
Q \equiv \frac{\lambda_{N}}{\lambda_{2}}<\frac{\alpha_{B}}{\alpha_{A}} .
$$

It is important to notice that the left hand side in the above inequality depends exclusively on the network topology, while the right hand side depends only on the dynamics (through $\mathbf{x}^{s}, \mathbf{F}$ and $\mathbf{H}$ ). The $\sigma$ range for which the synchronized state is stable, $\sigma \in\left[\frac{\alpha_{A}}{\lambda_{2}}, \frac{\alpha_{B}}{Q \lambda_{2}}\right]$, is larger for smaller eigenratios $Q$. In this way, networks with very small $Q$ will exhibit very good (robust) synchronization properties for a generic dynamics. The aim of this paper is to find and characterize network topologies minimizing the eigenratio $Q$.

\section{Optimizing Synchronizability: Entangled Networks}

Most studies up to now have explored the value of the eigenratio $Q$ for different pre-existing network topologies found in literature, as for instance small-world 


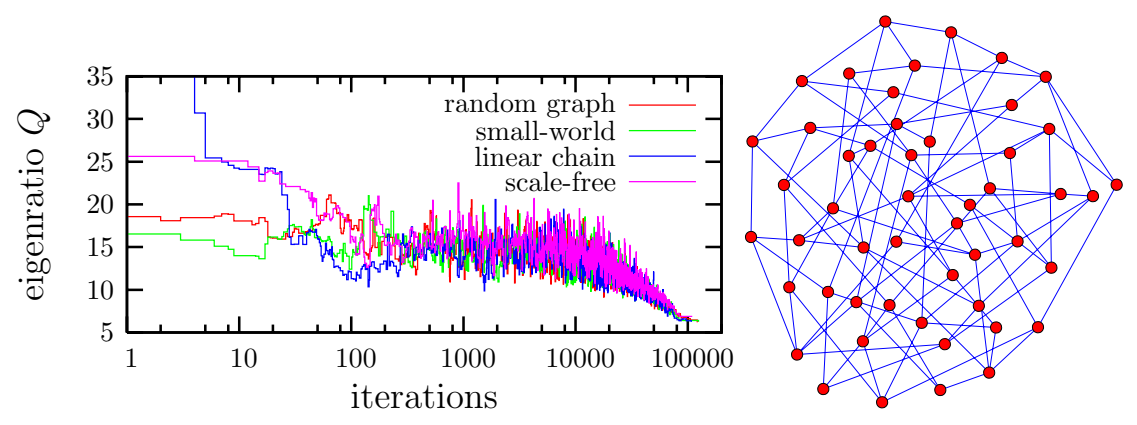

Fig. 1. Evolution of the eigenratio $Q$ during the optimization procedure for different initial conditions. Here $N=50,\langle k\rangle=4$. In all cases, the algorithm converges to very homogeneous networks as the one depicted, with very similar values of $Q$.

or scale-free networks, trying to identify key topological features affecting $Q$. In this way, it has been reported that small-worlds have smaller $Q$ than regular or purely random graphs [6], and this has been attributed to a smaller average distance between nodes in small-worlds. However, other works [8] have concluded recently that $Q$ decreases as some heterogeneity measures decrease, even if the average distance between nodes increases in the process. On the other hand, synchronizability is enhanced in weighted complex networks [9].

In this paper we undertake a constructive approach to determine the network topology that optimizes synchronization. In order to do so, we devise a modified simulated annealing algorithm [10] to numerically minimize $Q$. We start from graphs with $N$ nodes and a fixed average degree $\langle k\rangle$. At each step, a new graph is obtained by the random deletion of $m$ links and addition of $m$ new ones, where $m$ is extracted from an exponentially decaying distribution [7. The new graph is rejected if the resulting network is disconnected; otherwise, it is accepted with probability $p=\min \left(1,[1-(1-q) \delta Q / T]^{1 /(1-q)}\right)$, where $\delta Q=Q_{\text {final }}-Q_{\text {initial }}$ is the eigenratio change in the rewiring, and $T$ is a temperature-like parameter. For $q \rightarrow 1$ we recover the standard Metropolis algorithm with Hamiltonian $Q$, while $q=-3$ turns out to be the most efficient value (results do not depend on the choice of the deformation parameter $q$, but convergence times do [10]). The first $N$ rewirings are performed at $T=\infty$, and they are used to calculate a new $T$ such that the largest $\delta Q$ among the first $N$ ones would be accepted with a large probability. $T$ is kept fixed for $100 N$ rewiring attempts or $10 N$ accepted ones, whichever occurs first. Then $T$ is decreased by $10 \%$ and the process is repeated until there are no more changes during five successive temperature steps, assuming in this case that the optimal network topology has been found. Most of these details can be modified without affecting the final outcome. The major drawback in the algorithm is that $Q$ is a global observable slow to compute. For small enough $N(\leq 30)$, the emerging optimal topology found is unique, while for larger $N$ (we have optimized networks with $N$ up to 2000) the output may change slightly from run to run, meaning that the eigenratio absolute minimum is not always reached due to the presence of metastable states. Nevertheless, the 

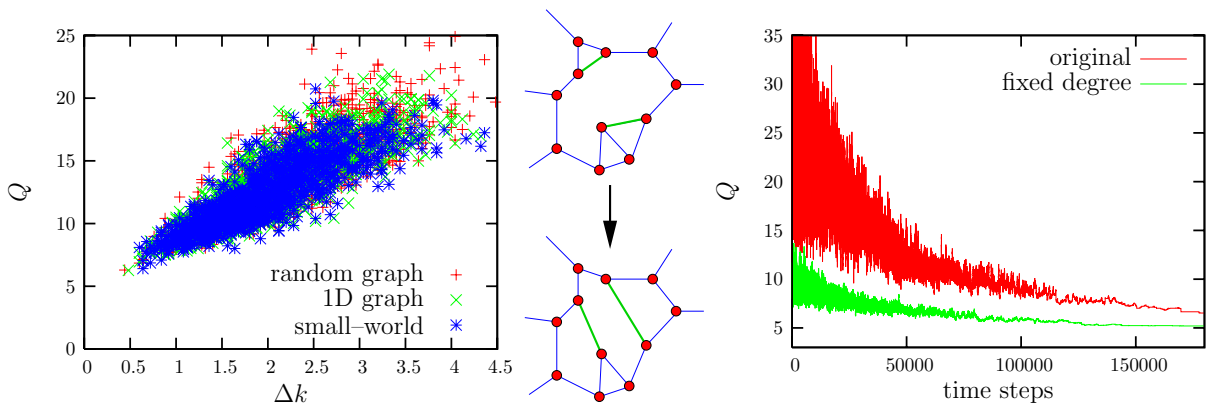

Fig. 2. Left: $Q$ vs standard deviation of the degree distribution for $N=50,\langle k\rangle=4$ and three different initial conditions. Center: sketch of pair rewiring trial. Right: Eigenratio $Q$ vs algorithmic steps for both minimization procedures (see text).

final values of $Q$ are very similar for different runs (see Fig. 1), meaning that a reasonably good approximation to the optimal topology is always found [7].

We measure different topological observables during the minimization process to unveil the main traits of the emerging structures. In simple terms, we observe that as $Q$ decreases the network becomes more and more homogeneous. This means that the standard deviation of distributions of most topological observables decreases as $Q$ decreases. This is true in particular for the node degree distribution, see Fig. 2, a. We have used this degree homogeneity to improve the efficiency our optimization procedure by initializing the algorithm with regular networks (i.e. all nodes with the same degree), and restricting the rewiring steps to changes that leave the degree of each node unchanged (by randomly selecting pairs of links and exchanging their endpoints; see Fig. 2. b). The resulting algorithm converges much faster to the optimal network, and yields lower final eigenratios $Q$ when the original one get trapped in a metastable state (Fig 2, c).

In Figs. 3] a-b we show the standard deviation of the average node-to-node distance and average betweenness, respectively, versus $Q$ during an optimization run started from a random regular graph. Both observables exhibit the aforementioned tendency towards homogeneity. Particularly remarkable is the narrow betweenness distribution (Fig. 3.b), which is in marked contrast with the broad betweenness distributions observed in networks with strong community structure [11. In addition, the averaged distance and betweenness also tend to decrease with $Q$, though they are less sensitive than their corresponding standard deviations, see Figs. 3. c-d. Another key feature of the optimal structures is the absence of short loops. This can be characterized by the girth (length of the shortest loop) or, better, via the average size of the shortest loop passing through each node. This last magnitude is shown in Fig. 3.e, where it is evident that the optimal network has very large average shortest loops. In particular, the clustering coefficient is zero for the optimal nets since no triangles are present.

In general, we call the emerging optimal structures entangled networks: all sites are very much alike (strong homogeneity) and the links form a very 


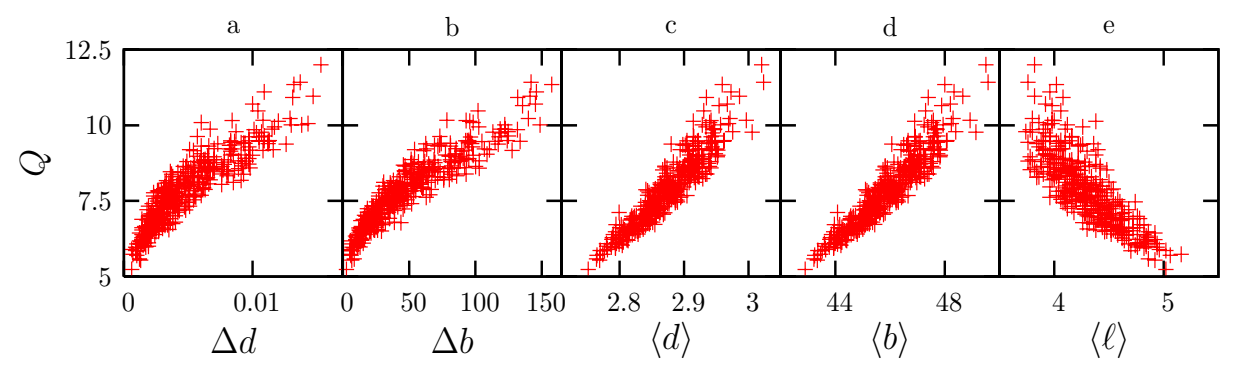

Fig. 3. Standard deviations of the node distance distribution (a), standard deviation of the betweenness distribution (b), average distance (c), average betweenness (d), and shortest-loop average length (e) as a function of the eigenratio $Q$

intricated or interwoven structure (lack of communities, poor modularity, and large shortest loops). Every single site is close to any other one (short average distances) owing not to the existence of intermediate highly connected hubs (as in scale free networks), but as a result of a very "democratic" or entangled structure in which properties such as site-to-site distance, betweenness, and minimum-loop size are very homogeneously distributed (see Figs. 1, b, 3, a-b). Sharp distributions are also typical of random graph, where randomness alone produces a statistical homogeneity. However in this case, a much stronger homogeneity is produced during the optimization of $Q$, as Figures 3 a-b show.

\section{Relation to Other Optimal Topologies}

A natural question concerns the relation between entangled networks and other optimal architectures found in the literature. For instance, recent work [12] has focused on the optimization of network robustness against random and/or intentional removal of nodes (attacks). For random graphs in the large- $N$ limit, it is concluded that the most robust networks are obtained when the degree distribution only has a few peaks. In particular, random $k$-regular graphs turn out to be the global optimal solution against both errors and attacks in the robustness-optimization problem [12. In this case, the error $\left(f_{r}\right)$ and attack $\left(f_{a}\right)$ percolation thresholds coincide, $f_{r}=f_{a} \equiv f_{c}(N, k)$, with $f_{c}(N, k)<f_{c}(\infty, k)=$ $(k-2) /(k-1)$. A natural question now is whether further $Q-$ minimization of these random regular graphs has some effect on the network robustness. As shown in [7] the minimization of $Q$ improves significantly the network robustness, confirming that entangled networks are optimal from the robustness point of view. This is because entangled topologies include correlations, absent in random networks, which enhance their resilience. In addition, there is also evidence that networks with properties similar to those of entangled graphs maximize reliability against link removal [7].

Different models of traffic flows on complex network have been recently studied [13, 14. In principle highly inhomogeneous scale-free networks perform well when the traffic is low; hubs can provide fast transition times, while they easily 
jam when the traffic increases. With the model of [14] it has been shown that if the density of traveling information packets is above a given threshold, the optimal network topology is a highly homogeneous, isotropic configuration, strongly resembling entangled graphs. In a similar way, it has been recently reported [15] that the interplay between network growth processes and evolutionary selection rules gives rise in some cases to very homogeneous structures with large minimal-loops that strongly resemble entangled networks (see Fig. 3.c in [15]).

Also, during our optimization procedure, $\lambda_{N}$ is observed to change very little with respect to $\lambda_{2}$, and therefore, minimizing $Q$ is equivalent for all practical purposes to maximizing $\lambda_{2}$. This provides another interesting connection with graph theory, where it is known that regular graphs with a large $\lambda_{2}$ (i.e.large spectral gap), are good expanders (see [16, 7] for a definition and applications). Expander graphs are very important in computer science for various applications (as the design of efficient communication networks or construction of error-correcting codes) and can be proved to exhibit a rapid decay of random-walk distributions towards their stationary state [17. This converts entangled graphs in (almost) optimal for many information flow processes.

\section{Summary and Outlook}

We have introduced the concept of "entangled networks" [7. These are constructed using an optimization principle by imposing the eigenvalues of the Laplacian matrix to have a quotient $\lambda_{N} / \lambda_{2}$ as small as possible, guaranteeing in this way a robust synchronizability and coherent behavior. The emerging topologies are extremely homogeneous: all nodes look very much alike (constituting a topology radically distinct from scale free networks). Also, the node-to-node average distance tends to be small while the average shortest loops are very large, and there is no modular (or community) structure. Entangled networks exhibit optimal synchronization properties, but they are also optimal or almost-optimal for other communication or flow properties: robustness and resilience against errors and attacks, traffic flow in the presence of congestion, relaxation properties of random walks, etc. These connections make of entangled networks a key tool in the context of complex networks.

An interesting issue concerns the existence of entangled networks in Nature. Their construction requires a global optimization process which is unlikely to occur in natural evolving systems. Presently, we are working on the identification of local evolutionary rules which give rise to locally-optimal synchronizable network patterns, or other feasible approximations to entangled networks.

\section{Acknowledgments}

We thank D. Cassi and P.L. Krapivsky for useful discussions, and B. Tadić and S. Thurner for inviting us to the ICCS06. Financial support from the Spanish MCyT under project No. FIS2005-00791, EU COSIN-project-IST2001-33555, and EU HPRN-CT-2002-00307 (DYGLAGEMEM) are also acknowledged. 


\section{References}

1. Barabási, A.-L.: Linked: The New Science of Networks, Perseus Publishing, Cambridge (2002); Pastor-Satorras, R., Vespignani, A.: Evolution and Structure of the Internet, Cambridge Univ. Press, Cambridge (2004).

2. Dorogovtsev, S.N., Mendes, J.F.F.: Evolution of Networks, Adv. Phys. 51, 10791187 (2002); Albert, R., Barabási, A.-L.: Statistical Mechanics of Complex Networks, Rev. Mod. Phys. 74, 47-97 (2002). Krapivsky, P.L., Redner, S.: A Statistical Physics Perspective on Web Growth, Computer Networks 39, 261-276 (2002).

3. Pikovsky, A., Rosenblum, M., Kurths, J.: Synchronization: A Universal Concept in Nonlinear Sciences, Cambridge University Press, Cambridge (2001); Glass, L.: Synchronization and Rhythmic Processes in Physiology, Nature 410, 277-284 (2001).

4. Belykh, I., de Lange, E., Hasler, M.: Synchronization of Bursting Neurons: What Matters in the Network Topology, Phys. Rev. Lett. 94, 188101 (2005); Cossart, R., Aronov, D., Yuste, R.: Attractor Dynamics of Network Up States in the Neocortex, Nature 423, 283-288 (2003).

5. Arenas, A., Díaz-Guilera, A., Pérez-Vicente, C.J.: Synchronization Reveals Topological Scales in Complex Networks, ArXiv cond-mat/0511730.

6. Barahona, M., Pecora, L.M.: Synchronization in Small-World Systems, Phys. Rev. Lett. 89, 054101 (2002); Pecora, L.M., Carrol, T.L.: Master Stability Functions for Synchronized Coupled Systems, Phys. Rev. Lett. 80, 2109-2112 (1998); Pecora, L.M., Barahona, M.: Synchronization of Oscillators in Complex Networks, Chaos and Complexity Letters, 1(1), 61-91 (2005).

7. Donetti, L., Hurtado, P.I., Muñoz, M.A.: Entangled Networks, Synchronization, and Optimal Network Topology, Phys. Rev. Lett. 95, 188701 (2005).

8. Nishikawa, T., et al. : Heterogeneity in Oscillator Networks: Are Smaller Worlds Easier to Synchronize?, Phys. Rev. Lett. 91, 014101 (2003); Hong,H., et al.: Factors that Predict Better Synchronizability on Complex Networks, Phys. Rev. E 69, 067105 (2004).

9. Chavez, M. et al. : Synchronization is Enhanced in Weighted Complex Networks, Phys. Rev. Lett. 94, 218701 (2005).

10. Penna, T.J.P.: Traveling Salesman Problem and Tsallis Statistics, Phys. Rev. E 51, R1-R3 (1995).

11. L. Donetti and M. A. Muñoz, Detecting Network Communities: a new systematic and powerful algorithm, J. Stat. Mech.: Theor. Exp. (2004) P10012-P10027.

12. Valente, A.X.C.N., Sarkar, A., Stone, H.A.: Two-Peak and Three-Peak Optimal Complex Networks, Phys. Rev. Lett. 92, 118702 (2004).

13. B. Tadić, S. Thurner, G. J. Rodgers: Traffic on complex networks: Towards understanding global statistical properties from microscopic density fluctuations, Phys. Rev. E, 69, 036102 (2004).

14. Guimerà, R., et al. : Optimal Network Topologies for Local Search with Congestion, Phys. Rev. Lett. 89, 248701 (2002).

15. Colizza, V. et al.: Network Structures from Selection Principles, Phys. Rev. Lett. 92, 198701 (2004).

16. Sarnak, P.: What is an Expander?, Not. Am. Math. Soc. 51, 762-763 (2004).

17. Lovasz, L.: Random Walks on Graphs: A Survey, in Combinatorics, Paul Erdös is Eighty, vol. 2, pp. 1-46, Keszthely, Hungary (1993). 